\title{
Interplay between sublattice and spin symmetry breaking in graphene
}

\author{
D. Soriano ${ }^{1,2}$ and J. Fernández-Rossier ${ }^{2,3}$ \\ ${ }^{1}$ CIN2 (ICN-CSIC) and Universitat Autonoma de Barcelona, Catalan Institute of Nanotechnology, \\ Campus UAB, 08193 Bellaterra (Barcelona), Spain \\ ${ }^{2}$ Departamento de Fisica Aplicada, Universidad de Alicante, San Vicente del Raspeig, Spain \\ ${ }^{3}$ International Iberian Nanotechnology Laboratory, Av. Mestre José Veiga, 4715-330 Braga, Portugal
}

(Received 17 January 2012; published 15 May 2012)

\begin{abstract}
We study the effect of sublattice symmetry breaking on the electronic, magnetic, and transport properties of two-dimensional graphene as well as zigzag terminated one- and zero-dimensional graphene nanostructures. The systems are described with the Hubbard model within the collinear mean field approximation. We prove that for the noninteracting bipartite lattice with an unequal number of atoms in each sublattice, in-gap states still exist in the presence of a staggered on-site potential $\pm \Delta / 2$. We compute the phase diagram of both $2 \mathrm{D}$ and $1 \mathrm{D}$ graphene with zigzag edges, at half filling, defined by the normalized interaction strength $U / t$ and $\Delta / t$, where $t$ is the first neighbor hopping. In the case of $2 \mathrm{D}$ we find that the system is always insulating, and we find the $U_{c}(\Delta)$ curve above which the system goes antiferromagnetic. In 1D we find that the system undergoes a phase transition from nonmagnetic insulator for $U<U_{c}(\Delta)$ to a phase with ferromagnetic edge order and antiferromagnetic interedge coupling. The conduction properties of the magnetic phase depend on $\Delta$ and can be insulating, conducting, and even half-metallic, yet the total magnetic moment in the system is zero. We compute the transport properties of a heterojunction with two magnetic graphene ribbon electrodes connected to a finite length armchair ribbon and we find a strong spin filter effect.
\end{abstract}

DOI: 10.1103/PhysRevB.85.195433

PACS number(s): 72.80.Vp, 72.25.Dc, 73.22.Pr

\section{INTRODUCTION}

The most salient electronic properties of graphene and its nanostructures are linked to the bipartite nature of the honeycomb lattice which is formed by two interpenetrating identical triangular sublattices. ${ }^{1}$ It is customary to refer to the sublattice as a pseudospin degree of freedom. In this language, the first neighbor hopping is described in terms of a pseudospin flip operator, which results in the well studied electron-hole symmetric bands in graphene, whose wave functions are sublattice unpolarized. The pseudospin symmetry becomes a chiral symmetry in the continuum limit in which electrons in graphene are described with a Dirac Hamiltonian $^{2}$ and accounts for the lack of backscattering, ${ }^{3}$ the so-called chiral tunneling, ${ }^{4}$ and the absence of an energy gap in two-dimensional graphene.

Sublattice symmetry breaking in graphene could arise spontaneously, due to some electronic phase transition, ${ }^{5-9}$ or due to the coupling of graphene to some substrate, like silicon carbide ${ }^{10,11}$ and boron nitride. ${ }^{12-14}$ Sublattice symmetry breaking would make it energetically favorable for the electrons to stay in one of the sublattices, resulting in pseudospin order ${ }^{15}$ (either spontaneous, or induced). The purpose of this work is to understand the interplay between induced pseudospin order and real spin order in graphene. Magnetic order is expected to take place in monohidrogenated graphene zigzag edges. Within the standard one-orbital tight-binding model of graphene, these edges give rise to a large density of states at the Fermi energy ${ }^{16}$ which is prone to a ferromagnetic instability when Coulomb repulsion is considered within the mean field Hubbard model. ${ }^{17}$ Density functional calculations confirmed the scenario, ${ }^{18,19}$ showing that the long-range Coulomb interactions and the other atomic orbitals, absent in the Hubbard model, do not play a major role in this system. Both the mean field Hubbard model ${ }^{17,20-27}$ and DFT calculations show that the magnetic phase with zero total spin has a gap, which opens due to interedge correlations. ${ }^{22}$ The fabrication of graphene ribbons with ultrasmooth edges is now possible by unzipping carbon nanotubes. ${ }^{28-30}$ Indirect evidence of magnetic order in the edges of zigzag ribbons is provided by scanning tunneling spectroscopy (STS) that can be accounted for within the mean field Hubbard model. ${ }^{31}$ Additional evidence is coming from magnetization and electron spin resonance experiments on unzipped graphene ribbons. ${ }^{32}$

The sublattice degree of freedom plays a central role in the magnetic properties of bipartite lattices. ${ }^{33-35}$ Very much like an external magnetic field favors one spin orientation and splits the spin states, an external perturbation favors one sublattice with respect to the other and opens a gap in the band structure of graphene. ${ }^{2,36}$ When this happens, it is not obvious a priori what happens to the edge states, even at the single particle level, and the associated magnetism. DFT calculations indicate that boron nitride $(\mathrm{BN})$ zigzag ribbons with the edge atoms passivated with hydrogen are nonmagnetic, ${ }^{37,38}$ whereas graphene ribbons, deposited on boron nitride whose lattice parameter is shifted to match graphene, indicate that edge magnetism survives. ${ }^{39}$ These DFT calculations suggest that as the sublattice symmetry breaking potential $\Delta$ increases, a phase transition must occur from magnetic to nonmagnetic edges. Here we address this problem using a much simpler description of the electron-electron interactions, namely, the mean field approximation for the Hubbard model, in the spirit of earlier work for graphene with the full sublattice symmetry and on recent work for graphene zigzag ribbons without inversion symmetry. ${ }^{40}$

The rest of this paper is organized as follows. In Sec. II we present some general theorems regarding the properties of the single particle states of the tight-binding model for graphene with a staggered potential. In Sec. III we study the 
interacting model for the case of two-dimensional graphene and study how the staggered potential affects the nonmagnetic to antiferromagnetic transition. In Sec. IV we study the interplay of magnetic and pseudospin order in the case of zigzag ribbons. We find that magnetic order and sub blattice symmetry breaking give rise to spin polarization of the bands, and in some instances we find half-metallic antiferromagnetic order. The spin filter properties of this case are studied in Sec. V, where we consider quantum transport between two half-metallic zigzag ribbons separated by a nonmagnetic armchair central region. In Sec. VI we summarize our main findings.

\section{SINGLE PARTICLE STATES OF A BIPARTITE LATTICE WITH A STAGGERED POTENTIAL}

In this section we consider some quite general properties of the single particle states of the Hamiltonian of a bipartite sublattice with a constant sublattice symmetry breaking term:

$$
\mathcal{H}_{0}=\left(\begin{array}{cc}
0 & h_{A B} \\
h_{B A} & 0
\end{array}\right)+\frac{\Delta}{2}\left(\begin{array}{cc}
\mathbf{1} & 0 \\
0 & \mathbf{- 1}
\end{array}\right)=h_{0}+V,
$$

where $h_{A B}, h_{B A}$, and $\mathbf{1}$ are matrices with dimension given by the number of atoms in sublattices $A$ and $B$.

\section{A. Null sublattice imbalance}

We consider first the case of a bipartite lattice without sublattice imbalance, so that the number of atoms in sublattice $A$ equals those in lattice $B: N_{A}=N_{B}$. In that case, $h_{A B}$, $h_{B A}$, and 1 are all matrices of range $N_{A}=N_{B}$. It can be easily seen that the sublattice symmetric Hamiltonian $h_{0}$ (or unperturbed Hamiltonian) anticommutes with the sublattice imbalance operator $\sigma_{z}$ :

$$
\left[h_{0}, \sigma_{z}\right]_{+} \equiv\left[\left(\begin{array}{cc}
0 & h_{A B} \\
h_{B A} & 0
\end{array}\right),\left(\begin{array}{cc}
1 & 0 \\
0 & -1
\end{array}\right)\right]_{+}=0 .
$$

Since $\sigma_{z}^{2}=1$, it is said that the graphene Hamiltonian has a chiral symmetry. As a result, if $\vec{\psi} \equiv\left(\begin{array}{c}\vec{\psi}_{A} \\ \vec{\psi}_{B}\end{array}\right)$ is an eigenstate of $h_{0}$ with energy $E$, we automatically have that $\vec{\phi} \equiv \sigma_{z} \vec{\psi}$ is also an eigenstate with energy $-E$ :

$$
h \vec{\psi}=E \vec{\psi}, \rightarrow h \vec{\phi}=-E \vec{\phi} .
$$

Thus, the chiral symmetry ensures that the spectrum of $h_{0}$ has electron-hole symmetry. In addition, since $\vec{\psi}$ and $\vec{\phi}$ are eigenvectors of the same Hamiltonian with different eigenvalues, they must be orthogonal. This leads to

$$
0=\vec{\psi}^{*} \cdot \vec{\phi}=\left\langle\vec{\psi}\left|\sigma_{z}\right| \vec{\psi}\right\rangle
$$

or more explicitly

$$
0=\sum_{i \in A}\left|\psi_{A}(i)\right|^{2}-\sum_{j \in B}\left|\psi_{B}(j)\right|^{2} .
$$

Thus, eigenstates of $h_{0}$ have the same weight on the two sublattices. In a pseudospin language, they have a zero expectation value of the $\sigma_{z}$ pseudospin operator, since the Hamiltonian has the pseudomagnetic field (the hopping) in the $x, y$ plane.
We now turn our attention to the eigenstates of a tightbinding Hamiltonian with first neighbor hoppings defined in a bipartite lattice with a sublattice-dependent potential which is both homogeneous and traceless, as defined by equation (1). We are going to show that they also have electron-hole symmetry. For that matter, we represent $\mathcal{H}_{0}$ in the subspace defined for a pair of eigenstates of $h_{0}, \vec{\psi}$, and $\vec{\phi}=\sigma_{z} \vec{\psi}$, with energies $E$ and $-E$, respectively. Using their definition and equation (5) we obtain

$$
\mathcal{H}_{0}=\left(\begin{array}{cc}
E & 0 \\
0 & -E
\end{array}\right)+\frac{\Delta}{2}\left(\begin{array}{ll}
0 & 1 \\
1 & 0
\end{array}\right),
$$

whose eigenvalues are $\epsilon_{ \pm} \equiv \pm \sqrt{E^{2}+\frac{\Delta^{2}}{4}}$ with corresponding eigenvectors $\vec{v}_{ \pm}$given by

$$
\vec{v}_{+}=\cos \frac{\theta}{2} \vec{\psi}+\sin \frac{\theta}{2} \vec{\phi}
$$

and

$$
\vec{v}_{-}=\sin \frac{\theta}{2} \vec{\psi}-\cos \frac{\theta}{2} \vec{\phi},
$$

where $\cos \theta=\frac{E}{\sqrt{E^{2}+\frac{\Delta^{2}}{4}}}$. Thus, if we know (half of) the spectrum and the eigenstate of the sublattice symmetric problem $h_{0}$, we can easily build the spectrum and the eigenfunctions for the same lattice when a homogeneous traceless sublattice Zeeman term is added to the Hamiltonian.

\section{B. System with sublattice imbalance}

The results of the previous section need to be examined with care in the special case that $E=0$. This certainly happens when we consider a system with $N_{A}=N_{B}+N_{Z}$, where $N_{Z}>$ 0 is a positive integer. In that case the dimension of the $A$ and $B$ subspaces is not the same, and the results of the previous section do not hold in general. ${ }^{41}$ In particular, it has been shown that $h_{0}$ has $N_{Z}$ eigenstates $\vec{\psi}_{Z}$ with $E=0$ that are sublattice polarized in the majority sublattice, $\vec{\psi}_{Z}=\left(\begin{array}{c}\vec{z}_{A} \\ 0\end{array}\right)$. These are the so-called midgap states that play a crucial role in the emergence of magnetism in graphene zigzag edges ${ }^{17}$ and graphene with chemisorbed hydrogen. ${ }^{42}$

It can be immediately seen that if $\vec{\psi}_{Z}$ is a zero energy eigenstate of $h$, it is also an eigenstate of $\mathcal{H}=h_{0}+V$ with eigenvalue $\epsilon=+\frac{\Delta}{2}$. Conversely, if $h_{0}$ presents a zero energy state sublattice polarized in $B$, then that state is also an eigenvector of $\mathcal{H}_{0}$ with energy $-\frac{\Delta}{2}$. This result was also found by Pereira et al. ${ }^{41}$

Thus, we can now predict the evolution of the spectrum of a given system described by $\mathcal{H}_{0}$ that, when $\Delta=0$, has midgap states at zero energy as well as pairs of electron-hole symmetric states with finite energies $\pm E_{n}$. The midgap states will become in-gap states with energy $\pm \Delta$, depending on their sublattice polarization, and the finite energy states will evolve as

$$
\epsilon_{ \pm}(\Delta)= \pm \sqrt{E_{n}^{2}+\frac{\Delta^{2}}{4}} .
$$

In order to illustrate this result, we have computed the single particle spectrum of a triangulene ${ }^{35}$ with $N_{A}=7$ and $N_{B}=$ 6 atoms. The evolution of the spectrum as a function of $\Delta$ 


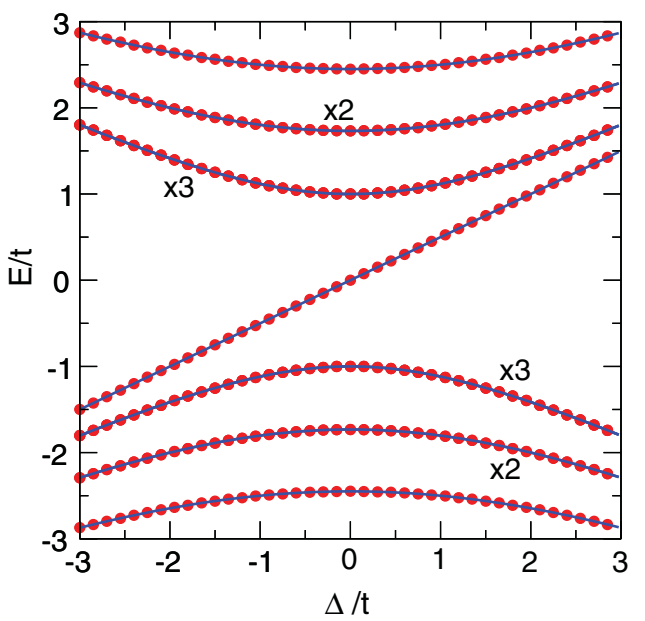

FIG. 1. (Color online) (a) Symbols: energy levels for triangulene with $N=13$ atoms calculated by diagonalization of the singleparticle model. Lines: energy levels obtained from equation (9). The degeneracies are indicated in the figure.

is shown in Fig. 1. We compare the result of the numerical diagonalization with those extrapolated from the spectrum of $h_{0}$ and, expectedly, find perfect agreement. This calculation shows that, in structures with a larger number of midgap states, the midgap shell will remain half full (when counting the spin), and interactions are expected to favor configurations with large spin.

\section{ELECTRONIC PROPERTIES OF TWO-DIMENSIONAL GRAPHENE WITH A STAGGERED POTENTIAL}

We now study the interplay between Coulomb repulsion and sublattice symmetry breaking. We model the interaction using a Hubbard model in the mean field approximation. For symmetric graphene this approximation is known to predict a phase transition from the nonmagnetic gapless state to an antiferromagnetic insulating state when ${ }^{43} U>U_{c}=2.2 t$. As usual, the mean field approximation underestimates the critical $U$ necessary for the Mott transition. Quantum Monte Carlo calculations indicate ${ }^{43}$ that the transition takes place at $U_{c} \simeq 4.5 t$. In addition, recent work $^{44}$ indicates that there might be a third phase with spin-liquid properties separating the nonmagnetic state from the magnetically ordered phase. In spite of its limitations, the mean field description of the Hubbard model can shed some light on the possible ordered phases and their electronic properties.

\section{A. Hubbard model and a mean field approximation}

The extended Hubbard model reads:

$$
\begin{aligned}
H & =t \sum_{i i^{\prime}, s} c_{i s}^{\dagger} c_{i^{\prime} s}+\frac{\Delta}{2} \sum_{i, s} \tau_{z}(i) c_{i s}^{\dagger} c_{i s}+U \sum_{i} n_{i \uparrow} n_{i \downarrow} \\
& =H_{0}+U \sum_{i} n_{i \uparrow} n_{i \downarrow},
\end{aligned}
$$

where $c_{i s}^{\dagger}$ creates an electron in atomic site $i$ with spin $s=$ $\uparrow, \downarrow, i^{\prime}$ stands for the first neighbors of $i$, and $\tau_{z}(i)=+1$ if $i$ belongs to the $A$ sublattice and -1 otherwise. We only consider the half-filling case, where the number of electrons equals the number of sites in the lattice, and we take $U>0$. For a given filling, the ground state properties of the model depend on two dimensionless parameters $\Delta / t$ and $U / t$. We explore the properties for a spin-collinear mean field approximation, where the interaction term is approximated by

$$
V_{M F}=U \sum_{i} n_{i \uparrow}\left\langle n_{i \downarrow}\right\rangle+\left\langle n_{i \uparrow}\right\rangle n_{i \downarrow},
$$

where $\left\langle n_{i s}\right\rangle$ is the average of the occupation operator of site $i$ with spin $s$, calculated with the many-body ground state of the mean field Hamiltonian:

$$
\left\langle n_{i s}\right\rangle=\sum_{\alpha} f_{\alpha}\left\langle\alpha\left|n_{i s}\right| \alpha\right\rangle,
$$

where $f_{\alpha}=0,1$ is the occupation of the single particle states $|\alpha\rangle$ that diagonalize calculated with the ground state of the mean field Hamiltonian $H_{0}+V_{M F}$. Since the potential $V_{M F}$ depends on the eigenstates of $H+V_{M F}$, both the potential and the eigenstates need to be computed self-consistently. We do this by iteration.

\section{B. Mean field approximation for 2D graphene}

We now describe the electronic properties of the Hubbard model for the two-dimensional honeycomb lattice with a staggered potential within the mean field approximation. In this case, we can take a minimal unit cell with 2 atoms, $A$ and $B$, and assume that the mean field in all unit cells is identical, which permits us to use Bloch theorem to represent the mean field Hamiltonian in the basis set $A \uparrow, B \uparrow, A \downarrow, B \downarrow$ :

$$
H=\left(\begin{array}{ll}
H_{\uparrow} & 0 \\
0 & H_{\downarrow}
\end{array}\right),
$$

where each element is a 2 by 2 matrix:

$$
H_{\uparrow}=\left(\begin{array}{lc}
\frac{\Delta}{2}+U\left\langle n_{A \downarrow}\right\rangle & f(\vec{k}) \\
f^{*}(\vec{k}) & -\frac{\Delta}{2}+U\left\langle n_{B \downarrow}\right\rangle
\end{array}\right)
$$

and

$$
H_{\downarrow}=\left(\begin{array}{lc}
\frac{\Delta}{2}+U\left\langle n_{A \uparrow}\right\rangle & f(\vec{k}) \\
f^{*}(\vec{k}) & -\frac{\Delta}{2}+U\left\langle n_{B \uparrow}\right\rangle
\end{array}\right)
$$

and

$$
f(k)=t\left(1+e^{i \vec{k} \cdot \vec{a}_{1}}+e^{i \vec{k} \cdot \vec{a}_{2}}\right)
$$

accounts for the first neighbor particle hopping. In our numerical determination of the self-consistent occupations $\left\langle n_{i s}\right\rangle$ we have taken a unit cell of four atoms. We have verified that our mean field solutions in this extended unit cell do not present intercell modulations of the charge density.

We have explored the phase diagram defined by $U / t$ and $\Delta / t$ and we find three types of solution, shown in Fig. 2:

(i) For $U<U_{c}(\Delta)$ the system is nonmagnetic and, except for $\Delta=0$, a band insulator. The case of $\Delta=0$ and $U<U_{c}$ is the well studied paramagnetic semimetal phase.

(ii) For $U>U_{c}(\Delta)$ the system is an antiferromagnetic insulator. The lack of inversion symmetry produced by the 

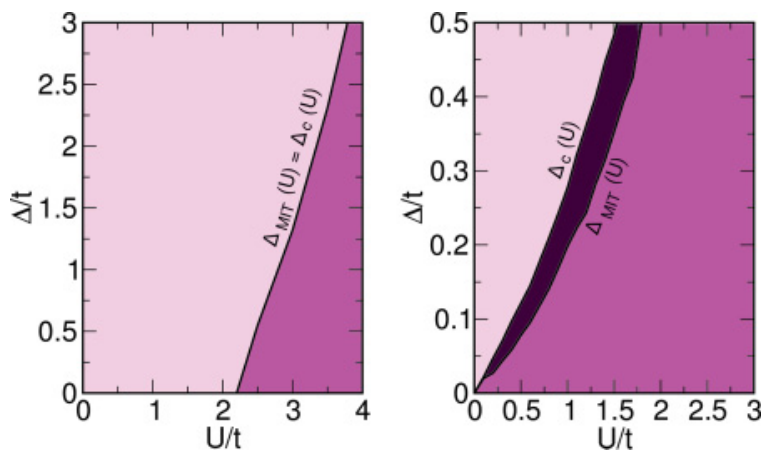

FIG. 2. (Color online) Phase diagrams for 2D graphene (left) and a zigzag graphene nanoribbon with $N=48$ atoms in the unit cell (right) with stagger potential $(\Delta)$ using a mean field Hubbard model at half filling. The dark region with $\Delta_{\mathrm{MIT}}(U)>\Delta(U)>\Delta_{c}(U)$ corresponds to the spin half-metallic phase in the graphene ribbon. In the case of $2 \mathrm{D}$ graphene, this region is reduced into a single critical line separating nonmagnetic and antiferromagnetic insulating states.

sublattice symmetry breaking results in a splitting of the spin bands, in contrast with the standard $\Delta=0$ case. This is shown in Fig. 3.

(iii) For $U=U_{c}(\Delta)$ the system is a half-semimetallic antiferromagnet. For one spin channel the system is insulating and for the other is semimetallic.

It is apparent that, as $\Delta$ increases, the critical $U_{c}$ increases. Expectedly, the magnetic order has to overcome the singleparticle gap opened by the staggered potential. Interestingly, the mean field approximation describes a magnetic transition between two insulating states, the nonmagnetic insulator and the antiferromagnetic insulating phase, which can be interpreted as an excitonic insulator transition. Given the large values of $U_{c}(\Delta) / t$, this ordered electronic phase is not expected in graphene. The predictions of this theory should be tested in cold atomic gases confined in optical lattices ${ }^{45}$ or in artificially patterned honeycomb lattices in two-dimensional electron gases. ${ }^{46,47}$

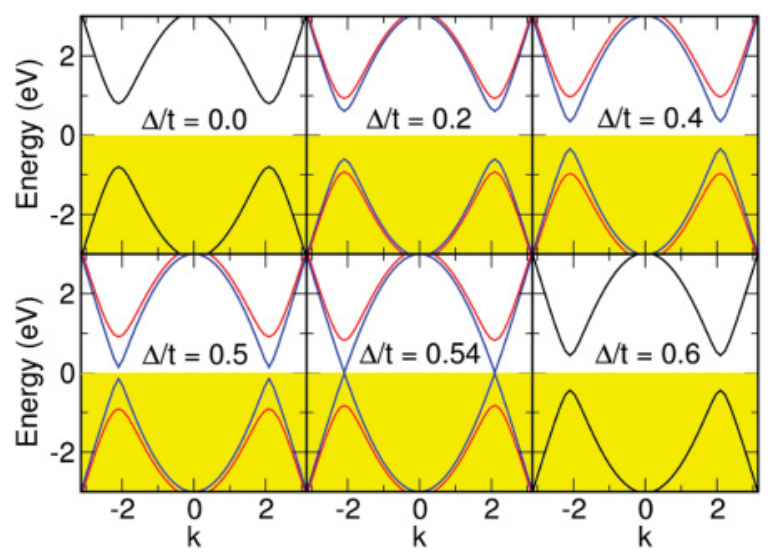

FIG. 3. (Color online) Evolution of the mean field bands for 2D graphene, for a fixed value of $U>U_{c}(\Delta=0)$ as $\Delta$ increases from 0 above $\Delta_{c}$.

\section{ELECTRONIC PROPERTIES OF GRAPHENE ZIGZAG RIBBONS WITH A STAGGERED POTENTIAL}

We now study the case of zigzag graphene ribbons for which we find that magnetic order could happen at small values of $U / t$ even for finite $\Delta$. The width of the ribbons is characterized by $N$, the number of atoms in the unit cell. Importantly, one of the edges is formed with $A$ atoms only, the other being made of $B$ atoms only. Thus, pseudospin polarization implies charge accumulation in one edge and depletion in the other, i.e., the formation of an electric dipole.

The electronic structure of graphene ribbons has been widely studied in the $\Delta=0$ limit, both for the $U=0^{16,48}$ and the finite $U$ cases. ${ }^{17-22,25,27,49}$ The most prominent feature of their electronic structure is given by the flat bands associated to edge states. At $\Delta=U=0$, these bands are located at the Fermi energy, giving rise to a large density of states at the Fermi energy. Not surprisingly, Coulomb repulsion results in a magnetic instability ${ }^{17}$ corresponding to the formation of magnetic moments in both edges while the bulk atoms remain almost spin unpolarized. It turns out that the interedge spin correlations are antiferromagnetic, as expected from the Lieb theorem. ${ }^{33}$ Thus, for a given spin orientation, there is charge accumulation in one of the edges and charge depletion in the opposite. This results in a spin-resolved pseudospin polarization, or spin dipole. ${ }^{22}$ Here we are interested in the interplay between pseudospin polarization, driven by the $\Delta$ term in the Hamiltonian, and the spin polarization, which entails a spin-resolved pseudospin polarization, driven by the Coulomb repulsion $U$.

\section{A. Noninteracting bands}

We first review the effect of the staggered potential on the noninteracting bands, studied by Qiao et al. ${ }^{39}$ At $\Delta=0$ two almost flat bands, associated to edge states, lie at the Fermi energy. As $\Delta$ becomes finite (and positive), the bands at the $A$ edge are red-shifted and those at the $B$ edge are blue-shifted, resulting in a band-gap opening. This is seen in Fig. 4 for two ribbons with $N=40$ and $N=80$ atoms, for $\Delta=0$ (left columns) and $\Delta=0.2 t$ (right column). We also notice the low energy bands are quite similar for the $N=40$ and $N=80$ ribbons, whereas the gap between the higher energy bands is reduced for the wider ribbon. This is consistent with the fact that the lowest energy bands are edge states, relatively insensitive to the width of the ribbon, in contrast with higher energy bands made of quantum confined bulk states. ${ }^{48}$

Thus, for finite $\Delta$ and $U=0$, graphene zigzag ribbons are band insulators with pseudospin polarization that features two flat bands corresponding to the highest occupied and lowest unoccupied bands. For $\Delta>0$, the bands corresponding to both $\uparrow$ and $\downarrow$ spins in the $B$ edge are occupied, whereas those in edge $A$ are empty. As we show now, these bands are prone to magnetic instability, not unlike in the case with $\Delta=0$.

\section{B. Effect of Coulomb repulsion}

We study now the interplay between pseudospin polarization and Coulomb repulsion. For that matter, we use again the mean field approximation for the Hubbard model, as described in previous work. ${ }^{17,20,22-27,40}$ Numerically found solutions 

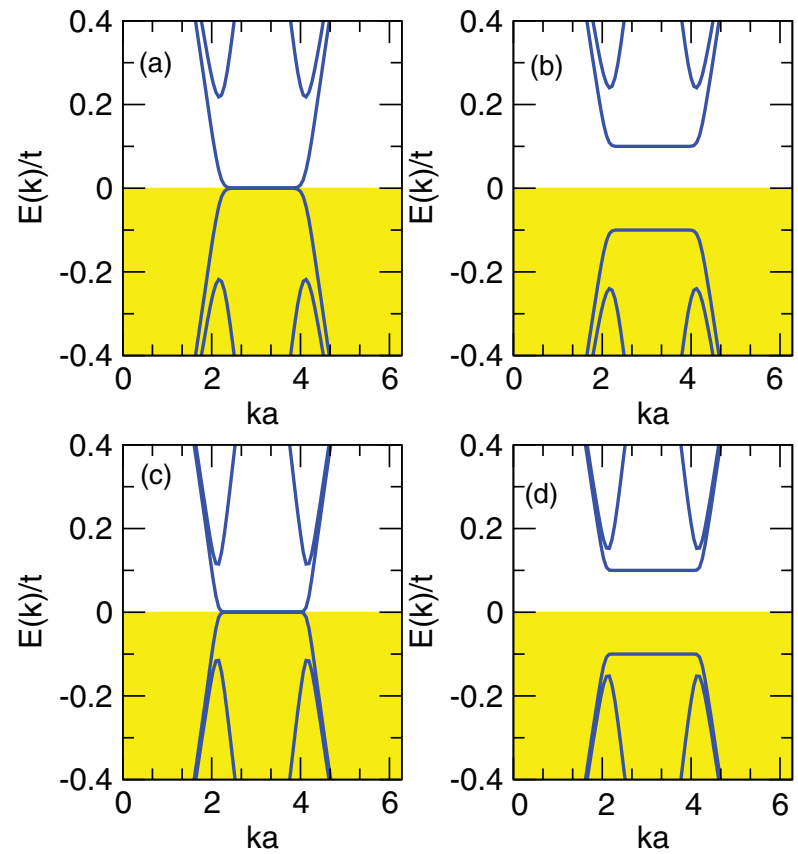

FIG. 4. (Color online) Electronic structure of zigzag ribbons with $U=0$, for $\Delta=0$ and $\Delta=0.2 t$ (left and right panels) for two different ribbon widths, $N=40$ (top) and $N=80$ (bottom). For the sake of clarity, we only plot the two higher energy valence bands and the two lowest energy conduction bands.

present magnetization at both edges equal in magnitude and opposite in sign. Thus, there are two equivalent ground states: $m_{A}=-m_{B}>0$ and $m_{A}=-m_{B}<0$. The corresponding energy bands for the ribbon with $N=48$, and $\Delta=0$ and $U=t$, are shown in Fig. 5(a). The magnetic order results in a band-gap opening. The spin $\uparrow$ and $\downarrow$ bands are degenerate. The magnetic moment at the edge atoms is $m= \pm 0.13$. The charge per atom is the same all over the unit cell, one electron per atom.

When the sublattice-symmetry breaking potential is finite and below a critical value $\Delta_{c}(U)$, we still find magnetic order in the edges with antiferromagnetic coupling, and zero total moment, even if the charge is no longer the same for both edges. The electronic properties of the magnetic ribbon with pseudospin polarization are different on several counts. First, the bands are spin split, as a natural consequence of the lack of both time-reversal and inversion symmetries. The evolution of the energy bands, as we increase $\Delta$, is shown in Fig. 5 for the $N=48$ ribbon with $U=t$. This figure can be understood as follows. The solution has $m_{A}=-m_{B}>0$ so that the $\uparrow$ band is occupied (empty) in the $A(B)$ edge. Conversely, the $\downarrow$ band is occupied (empty) in the $B(A)$ edge. As $\Delta$ is turned on, the $B$ bands are red-shifted and the $A$ bands are blue shifted. For the $\uparrow$ bands, this implies that the band gap closes, since the valence $A$ bands move upward and the conduction $B$ bands move downward. Conversely, the gap opens in the $\downarrow$ channel.

As shown in Fig. 6 and discussed below, as $\Delta$ increases the magnetic moment at the edges are depleted and eventually disappear when $\Delta>\Delta_{c}(U)$. Remarkably, the gap in the $\uparrow$ channel closes for $\Delta_{\mathrm{MIT}}(U)<\Delta_{c}(U)$, yet the gap is finite in the $\downarrow$ channel. Thus, the combination of pseudospin
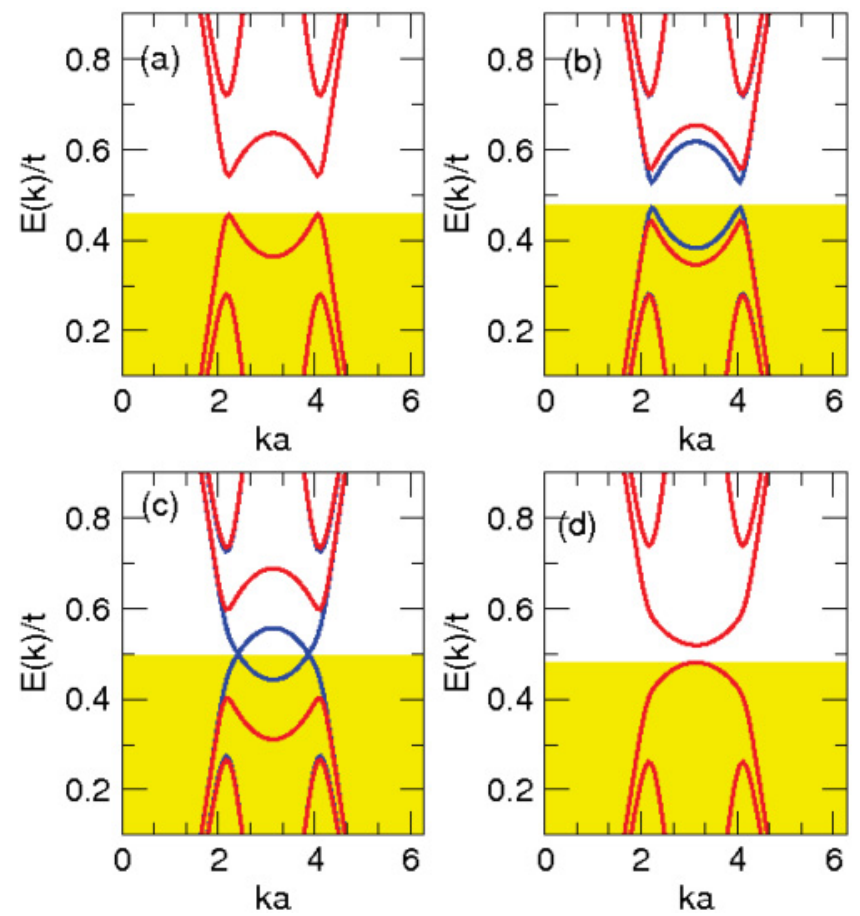

FIG. 5. (Color online) Lowest energy bands for zigzag ribbon with $N=48$ atoms in unit cell and $U=t$, for different values of $\Delta$ : (a) $\Delta=0$, (b) $\Delta=0.05 t$, (c) $\Delta=0.2 t$, (d) $\Delta=0.3 t$. Only the two highest energy occupied bands and the two lowest energy empty bands, per spin channel, are shown. Blue (red) stands for $\uparrow(\downarrow)$ bands.

polarization and antiferromagnetic order makes the system a half-metallic antiferromagnet in a region of the $\Delta, U$ phase space (see dark-violet region in the right panel of Fig. 2. Notice that this is different from the ferromagnetic half-metallic phase predicted for graphene ribbons in the presence of a transverse electric field, ${ }^{19}$ for which the total magnetic moment is different from zero.

Finally, we note that the gap of the nonmagnetic case in Fig. 5(d) for $\Delta>\Delta_{c}$ is significantly smaller than $\Delta$. This is due to the renormalization of the bands due to Coulomb repulsion. Basically, the occupied bands are blue shifted with respect to the empty bands, reducing the size of the gap.

\section{Phase Diagram}

In Fig. 2 we show the phase diagram defined by $U / t$ and $\Delta / t$ for a ribbon with $N=48$ atoms, calculated within the mean field approximation at half filling. Earlier work ${ }^{40}$ has addressed the phase diagram defined by $\Delta$ and the electron density. The diagram in Fig. 2 has two phases regarding the magnetic order: nonmagnetic, for $\Delta>\Delta_{c}(U)$, and antiferromagnetic otherwise. The results are very similar for ribbons with different widths. In contrast with the 2D case, for $\Delta=0$ the critical $U$ for the edge is zero. This makes zigzag graphene ribbons suitable systems for the observation of magnetism in graphene and the possible effect of sublattice symmetry breaking more relevant. Expectedly, the critical $\Delta_{c}(U)$ is an increasing function of $U$, or in other words: the larger the single particle gap, the strongest the interaction $U$ required to drive the magnetic instability. Spin polarization requires promotion of electrons across the single particle gap, 

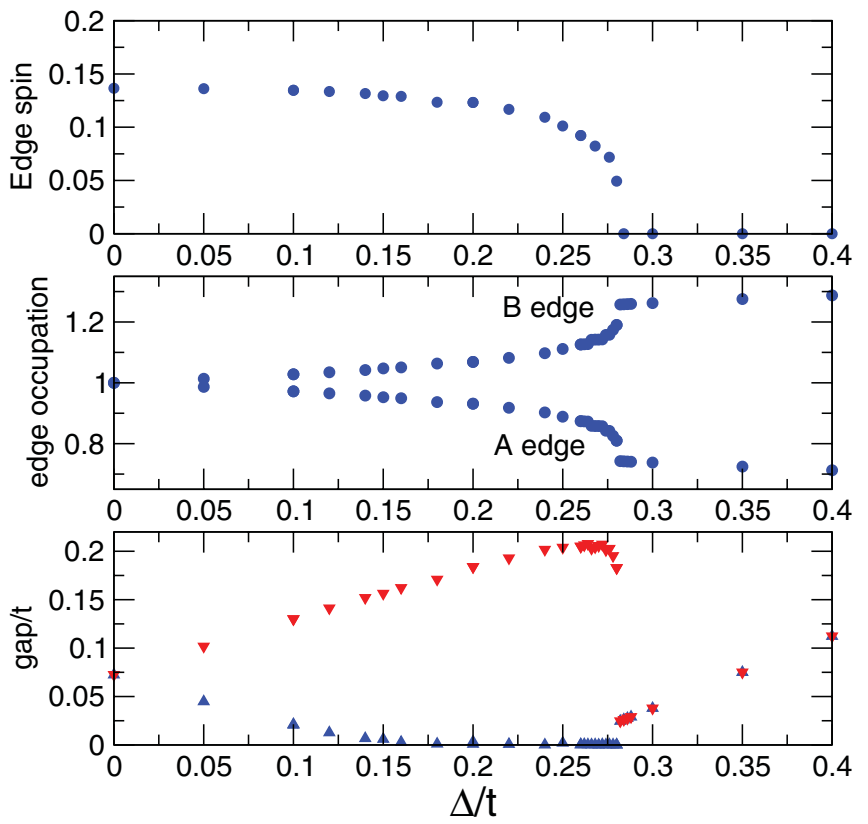

FIG. 6. (Color online) Properties of $N=48$ ribbon with $U=t$ as a function of $\Delta$. Top panel: edge magnetization. Middle panel: edge charges. Bottom panel: gap for spin $\uparrow$ and $\downarrow$.

from the occupied $B$ to the empty $A$ edge, i.e., the formation of a magnetic exciton condensate. The difference with the $\Delta=0$ case stands on the size of the the single particle gap, which is vanishingly small (but not zero) in finite width ribbons. $^{22}$ Interestingly, the magnetic exciton condensation scenario already takes place in the case apparently conducting $\Delta=U=0$ ribbon, when $U$ is turned on.

For a fixed value of $\Delta$, as the value of $U$ is increased the system undergoes a phase transition from a nonmagnetic band insulator, to a half-metallic antiferromagnet and then to an insulating antiferromagnet. The fact that interactions can drive the system from insulating to metallic is quite exotic and differs from the usual Mott insulator scenario, in which interactions drive a band metal insulating. In the phase diagram we mark the insulator to metal transition when the smallest energy gap, at a given spin channel, is 100 times smaller than the gap at $\Delta=0$. As shown in Fig. 6 for $U=t$, the gap at $\Delta=0$ is $0.07 t$. Thus, for that particular value of $U$, we declare the system conducting when the gap is below $7 \times 10^{-4} t$. Variations upon this criteria yield quantitative changes in the metal to insulator transition (MIT) line, but it is always the case that $\Delta_{\mathrm{MIT}}(U)$ runs along and below the magnetic phase transition line $\Delta_{c}(U)$.

\section{GRAPHENE ZIGZAG RIBBONS WITH STAGGERED POTENTIAL AS AN IDEAL SPIN INJECTOR}

Interestingly, the predicted conducting phase for $\Delta_{\mathrm{MIT}}(U)<\Delta<\Delta_{c}(U)$ is a half-metallic antiferromagnet that mimics the electronic properties of an ideal ferromagnetic spin injector. In this section we study the spin transport properties of a tunnel junction where the electrodes are made of such half-metallic antiferromagnets and the barrier is made of semiconducting armchair graphene ribbon.
In Fig. 7 we consider three possible situations, all of them with $\Delta=0.25 t, U=t$. The first and second cases feature two antiferromagnetic electrodes with mutually parallel and antiparallel magnetizations, respectively. The bands for the parallel case show a conducting and fully spin polarized character in an energy range $\sim 0.5 \mathrm{eV}$ around the Fermi energy which makes them suitable for spin injection in spintronics. Unlike the parallel case, we expect the tunnel conductance to be completely depleted when the magnetic moments of the different electrodes are antiparallel, mainly due to the opposite spin polarization of valence and conduction bands on each electrode. In the last case we consider two ferromagnetic electrodes. The bands of the infinite ribbon with ferromagnetic coupling between the magnetic edges reflect the conducting and spin unpolarized character of the system at the Fermi energy (set at $E=0 \mathrm{eV}$ in the three cases studied).

\section{A. Formalism}

The conductance $G$ of the system is calculated within the Landauer formalism for coherent quantum transport. In this approach we have $G(E)=\frac{e^{2}}{h} \operatorname{Tr}[T(E)]$ where $T(E)$ is the transmission function, $e$ is the charge of the electron, and $h$ is the Planck constant. The calculation of $T(E)$ requires the solution of a scattering problem for the Hamiltonian of two semiinfinite electrodes attached to the central region that describes the tunneling barrier. To compute the transmission function along the armchair nanoribbon we adopt a partition method as implemented in the ALACANT (Ant.U) ${ }^{50}$ transport package, where the system is divided into three parts, ${ }^{25,26}$ namely, the central $(C)$ part which contains both the nonmagnetic barrier and a short section of the zigzag ribbons, and the left and right semi-infinite staggered zigzag nanoribbons ( $L$ and $R$ ). The barrier is described with an armchair semiconducting graphene nanoribbon, including the mean field Hubbard term. The electrodes are two spin-polarized zigzag nanoribbons with both staggered and mean field Hubbard potentials, as those studied in the previous section. Except for the atoms at the interface with the zigzag ribbons, the Hubbard $U$ term is not able to spin polarize the armchair central region, as shown in Fig. 7. We neglect spin relaxation at the nonmagnetic barrier.

The transmission probability can then be obtained from

$$
T_{\sigma}(E)=\operatorname{Tr}\left[\mathcal{G}_{C}^{\dagger}(E) \Gamma_{R}(E) \mathcal{G}_{C}(E) \Gamma_{L}(E)\right]_{\sigma},
$$

where $\mathcal{G}_{C, \sigma}(E)=\left[z I-H_{C, \sigma}-\Sigma_{R, \sigma}(E)-\Sigma_{L, \sigma}(E)\right]^{-1}$ is the Green's function of the central region which contains all the information concerning the electronic structure of the semi-infinite leads through the self-energies $\left[\Sigma_{R, \sigma}(E)\right.$ and $\left.\Sigma_{L, \sigma}(E)\right]$, and $\Gamma_{R, \sigma}(E)=i\left[\Sigma_{L, \sigma}(E)-\Sigma_{L, \sigma}^{\dagger}(E)\right], \Gamma_{L, \sigma}(E)=$ $i\left[\Sigma_{R, \sigma}(E)-\Sigma_{R, \sigma}^{\dagger}(E)\right]$ are the coupling matrices containing the information about the coupling of the central region to the leads.

\section{B. Results}

In Figs. 8(a)-8(f), we show the results of the calculated spin-resolved conductance curves for the three systems shown in Fig. 7. The top panels [(a)-(c)] correspond to electrodes with finite $\Delta$, whereas middle panels [(d)-(f)] correspond to $\Delta=0$. In panels (a) and (b) both electrodes are antiferromagnetic 

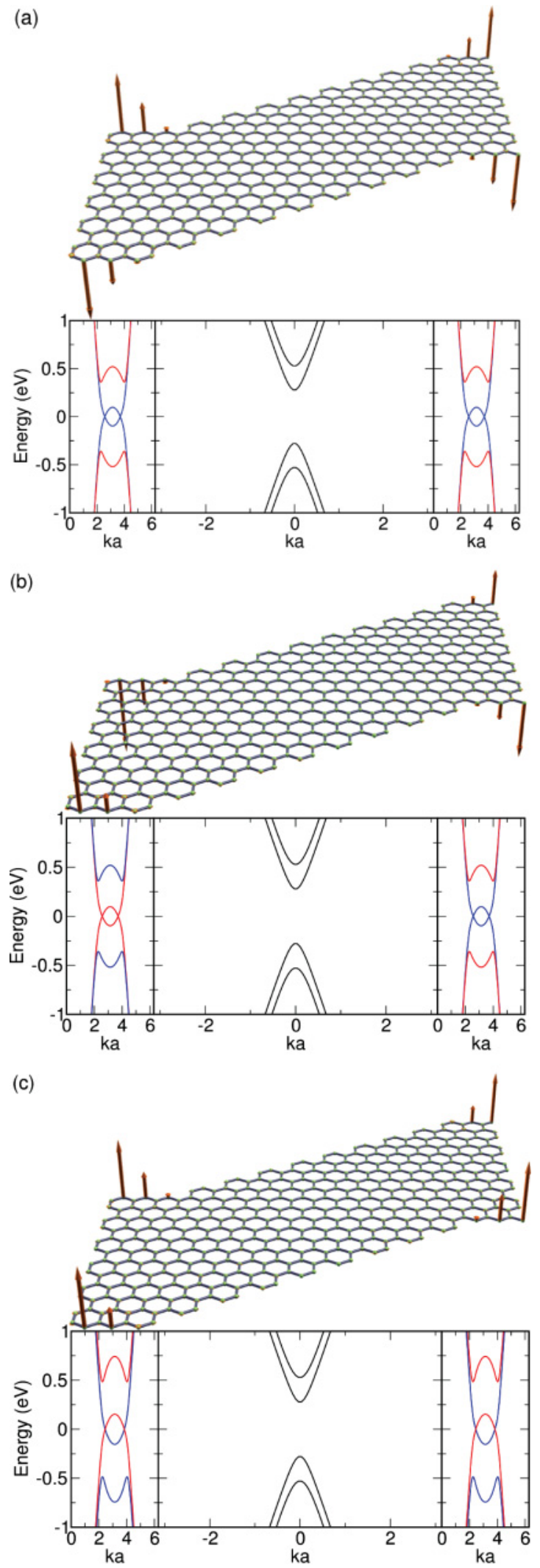

FIG. 7. (Color online) Band structure of the leads and central region for the three cases studied: (a) parallel antiferromagnetic electrodes, (b) antiparallel antiferromagnetic electrodes, and (c) parallel ferromagnetic electrodes. The central region is the same for the three cases studied, namely, a semiconducting armchair graphene nanoribbon with a local Coulomb potential $(U=t)$.

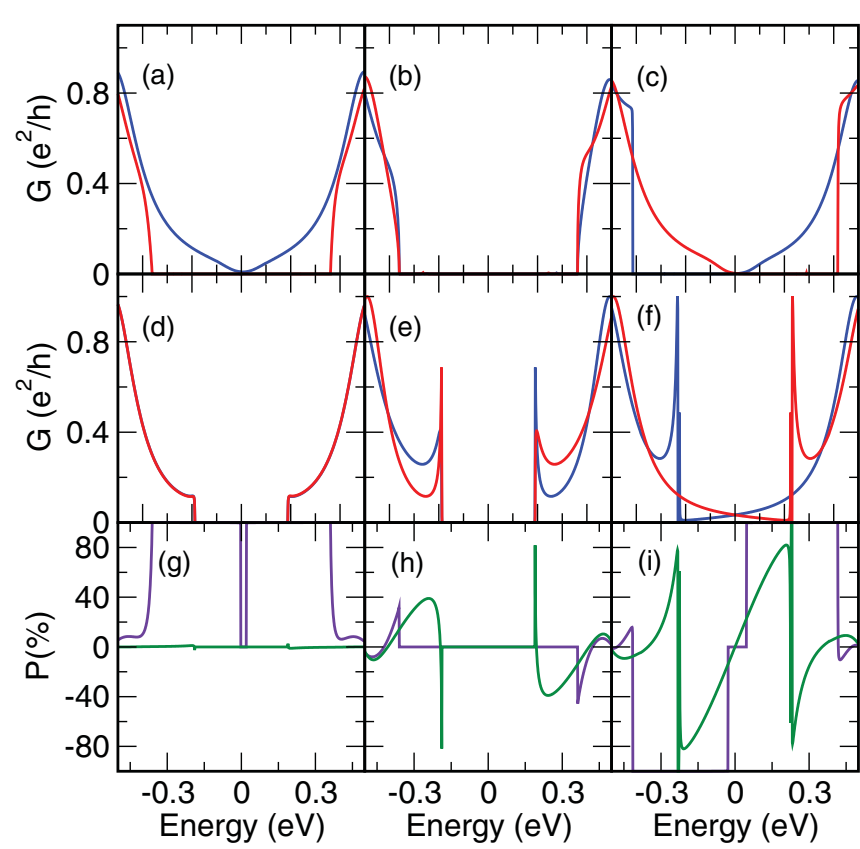

FIG. 8. (Color online) Spin conductance through a finite armchair ribbon connected to two spin-polarized zigzag ribbons with [(a)-(c)] and without $[(\mathrm{d})-(\mathrm{f})]$ stagger potential. The bottom panels [(g)-(i)] shows the spin conductance polarization for each magnetic ordering at the electrodes in the presence (violet) and absence (green) of stagger potential. The first and second columns correspond to the antiferromagnetic cases with mutual parallel and antiparallel configuration between leads, respectively. The third one corresponds to the ferromagnetic case.

half metals, with parallel (a) and antiparallel (b) relative spin orientations at the two sides of the barrier. Their half-metallic bands are shown in Figs. 7(a) and 7(b). The case with copolarized half-metallic AF electrodes [Fig. 8(a)] yields a tunnel current for the only spin channel available at the Fermi energy $(E=0)$. The other spin channel has strictly no conductance for $E$ close to 0 . As a result, the current is spin polarized. This is quantified by the conductance polarization $P(\%)=\left(\mathcal{G}_{\uparrow}-\mathcal{G}_{\downarrow}\right) /\left(\mathcal{G}_{\uparrow}+\mathcal{G}_{\downarrow}\right) \times 100$. This quantity is shown, at different energies, for $\Delta=0.25 t$ (violet line) and $\Delta=$ 0 (green line), in Figs. 8(g)-8(i). The case with counterpolarized half-metallic electrodes is shown in Fig. 8(b). As a result of the opposite spin polarization in the two electrodes, both spin channels are closed and we have $G=P=0$ close to $E=0$.

The case with finite $\Delta$ and interedge ferromagnetic correlations at both electrodes is shown in Figs. 7(c) and 8(c). In the ferromagnetic case, there is one open channel per spin at the electrodes, but the transmission properties are such that only one of them is open for transport. Thus, from the transport standpoint, the system behaves like a half metal. Thus, we have $P= \pm 1$, for $E \simeq 0$.

The middle panels [(d)-(f)] correspond to the case with $\Delta=0$ so that, for antiferromagnetic interedge correlation, the electrodes are insulating [not shown in Fig. (7)], and for ferromagnetic interedge correlation the electrodes are conducting. The two antiferromagnetic cases with $\Delta=0$ [Figs. 8(d) and 8(e)] show a large gap in the conductance and $P=0$ due to 
the semiconducting behavior of the three regions. ${ }^{18,22,25}$ For the ferromagnetic case with $\Delta=0$ [Fig. 8(f)], there is a finite conductance at the Fermi energy due to the metallic nature of the electrodes ${ }^{25}$ (bands not shown in Fig. 7 for this case). In this case, the evanescent modes coming from both electrodes penetrate into the central region and overlap due to the short length of the armchair ribbon. Accordingly, $P$ is finite, but smaller than in the case with finite $\Delta$.

\section{SUMMARY AND CONCLUSIONS}

Our main findings can be summarized as follows:

(i) We have shown that for a bipartite lattice with a siteindependent pseudospin Zeeman term the resulting spectrum has electron hole symmetry, except for midgap states that arise in lattices with a different number of sites in the two sublattices. We have shown that, if the $\Delta=0$ Hamiltonian has an eigenstate $\vec{\phi}$ with energy $E$, it has also an eigenstate $\vec{\psi}$ with energy $-E$, and the Hamiltonian with finite $\Delta$ has two eigenstates, a linear combination of $\vec{\phi}$ and $\vec{\psi}$ with energies $\pm \sqrt{\frac{\Delta^{2}}{4}+E^{2}}$.

(ii) If $\vec{\phi}$ localized in the $A(B)$ sublattice is an eigenstate with energy $E=0$ of the $\Delta=0$ Hamiltonian, then it is also an eigenstate of the Hamiltonian with finite $\Delta$ and energy $\Delta$ $(-\Delta)$.

(iii) Two-dimensional graphene with finite $\Delta$ undergoes a transition to an antiferromagnetic state with spin-split bands, for $U>U_{c}(\Delta)$. The critical $U_{c}$ is an increasing function of $\Delta$. At the transition between the nonmagnetic insulating state $\left[U<U_{c}(\Delta)\right]$ and the magnetic state $U>U_{c}$, the system is a half-metallic antiferromagnet.

(iv) Zigzag graphene ribbons with a finite sublattice symmetry-breaking potential below a critical value $[\Delta(U)<$ $\Delta_{c}(U)$ ] can undergo a transition from nonmagnetic insulators to spin-polarized half-metallic antiferromagnets in the presence of Coulomb interaction. The fact that interactions can drive the system from insulating to metallic differs from the usual Mott insulator scenario in which interactions drive a band metal insulating.

(v) Zigzag graphene ribbons with stagger potential $\Delta$ slightly below the $\Delta_{c}(U)$ are predicted to be ideal spin injectors. The spin transport calculations carried out in this work show high spin polarization of the conductance $(\approx 100 \%)$ around the Fermi level when used as spin injectors in a tunnel junction. This indicates that, if the suitable substrate that yields the right $\Delta$ is found, graphene zigzag ribbons could act as half-metallic spin injectors.

\section{ACKNOWLEDGMENTS}

This work has been financially supported by MECSpain (FIS- No. FIS2010-21883-C02-01, and CONSOLIDER CSD2007-0010). We acknowledge fruitful conversations with Jeil Jung and John W. González.
${ }^{1}$ A. H. Castro Neto, F. Guinea, N. M. R. Peres, K. S. Novoselov, and A. K. Geim, Rev. Mod. Phys. 81, 109 (2009).

${ }^{2}$ Gordon W. Semenoff, Phys. Rev. Lett. 53, 2449 (1984).

${ }^{3}$ T. Ando, T. Nakanishi, and R. Saito, J. Phys. Soc. Jpn. 67, 2857 (1998).

${ }^{4}$ M. I. Katsnelson, K. S. Novoselov, and A. K. Geim, Nat. Phys. 2, 620 (2006).

${ }^{5}$ H. Min, G. Borghi, M. Polini, and A. H. MacDonald, Phys. Rev. B 77, 041407(R) (2008).

${ }^{6}$ J. E. Drut and T. A. Lahde, Phys. Rev. B 79, 165425 (2009).

${ }^{7}$ Y. Araki, Phys. Rev. B 84, 113402 (2011).

${ }^{8}$ G. W. Semenoff, Phys. Scr. T 146, 014016 (2012).

${ }^{9}$ Y. Araki and G. W. Semenoff, e-print arXiv:1204.4531 (unpublished).

${ }^{10}$ S. Y. Zhou, G.-H. Gweon, A. V. Fedorov, P. N. First, W. A. de Heer, D.-H. Lee, F. Guinea, A. H. Castro Neto, and A. Lanzara, Nat. Mater. 6, 770 (2007).

${ }^{11}$ A. Bostwick, T. Ohta, J. L. McChesney, K. V. Emtsev, T. Seyller, K. Horn and E. Rotenberg, New J. Phys. 9, 385 (2007).

${ }^{12}$ G. Giovannetti, P. A. Khomyakov, G. Brocks, P. J. Kelly, and J. van den Brink, Phys. Rev. B 76, 073103 (2007).

${ }^{13}$ C. R. Dean, A. F. Young, I. Meric, C. Lee, L. Wang, S. Sorgenfrei, K. Watanabe, T. Taniguchi, P. Kim, K. L. Shepard, and J. Hone, Nat. Nanotechnol. 5, 722 (2010).

${ }^{14}$ J. Xue, J. Sanchez-Yamagishi, D. Bulmash, P. Jacquod, A. Deshpande, K. Watanabe, T. Taniguchi, P. Jarillo-Herrero, and B. J. LeRoy, Nat. Mater. 10, 282 (2011).
${ }^{15}$ A. H. MacDonald, J. Jung, and F. Fang, Phys. Scr. T 145, 014012 (2012).

${ }^{16}$ K. Nakada, M. Fujita, G. Dresselhaus, and M. S. Dresselhaus, Phys. Rev. B 54, 17954 (1996).

${ }^{17}$ M. Fujita, K. Wakabayashi, K. Nakada, and K. Kusakabe, J. Phys. Soc. Jpn. 65, 1920 (1996).

${ }^{18}$ Y.-W. Son, M. L. Cohen, and S. G. Louie, Phys. Rev. Lett. 97, 216803 (2006)

${ }^{19}$ Y.-W. Son, M. L. Cohen, and S. G. Louie, Nature (London) 444, 347 (2006).

${ }^{20}$ A. Yamashiro, Y. Shimoi, K. Harigaya, and K. Wakabayashi, Phys. Rev. B 68, 193410 (2003).

${ }^{21}$ D. Gunlycke, D. A. Areshkin, J. Li, J. W. Mintmire, and C. T. White, Nano Lett. 7, 3608 (2007).

${ }^{22}$ J. Fernández-Rossier, Phys. Rev. B 77, 075430 (2008).

${ }^{23}$ J. Jung, T. Pereg-Barnea, and A. H. MacDonald, Phys. Rev. Lett. 102, 227205 (2009).

${ }^{24}$ J. Jung and A. H. MacDonald, Phys. Rev. B 79, 235433 (2009).

${ }^{25}$ F. Muñoz-Rojas, J. Fernández-Rossier, and J. J. Palacios, Phys. Rev. Lett. 102, 136810 (2009).

${ }^{26}$ D. Soriano, F. Muñoz-Rojas, J. Fernández-Rossier, and J. J. Palacios, Phys. Rev. B 81, 165409 (2010)

${ }^{27}$ D. Soriano and J. Fernández-Rossier, Phys. Rev. B 82, 161302 (2010).

${ }^{28}$ X. Li, X. Wang, L. Zhang, S. Lee, and H. Dai, Science 319, 1229 (2008). 
${ }^{29}$ L. Jiao, X. Wang, G. Diankov, H. Wang, and H. Dai, Nat. Nanotechnol. 5, 321 (2010).

${ }^{30}$ X. Wang, Y. Ouyang, L. Jiao, H. Wang, L. Xie, J. Wu, J. Guo, and H. Dai, Nat. Nanotechnol. 6, 563 (2011).

${ }^{31}$ C. Tao, L. Jiao, O. V. Yazyev, Y.-C. Chen, J. Feng, X. Zhang, R. B. Capaz, J. M. Tour, A. Zettl, S. G. Louie, H. Dai, and M. F. Crommie, Nat. Phys. 7, 616 (2011).

${ }^{32}$ S. S. Rao, S. Narayana Jammalamadaka, A. Stesmans, V. V. Moshchalkov, J. van Tol, D. V. Kosynkin, A. Higginbotham-Duque, and J. M. Tour, Nano Lett. 12, 1210 (2012).

${ }^{33}$ Elliott H. Lieb, Phys. Rev. Lett. 62, 1201 (1989).

${ }^{34}$ L. Brey, H. A. Fertig, and S. Das Sarma, Phys. Rev. Lett. 99, 116802 (2007).

${ }^{35}$ J. Fernández-Rossier and J. J. Palacios, Phys. Rev. Lett. 99, 177204 (2007).

${ }^{36}$ Di Xiao, Wang Yao, and Qian Niu, Phys. Rev. Lett. 99, 236809 (2007).

${ }^{37}$ J. Nakamura, T. Nitta, and A. Natori, Phys. Rev. B 72, 205429 (2005).

${ }^{38}$ A. Lopez-Bezanilla, J. Huang, H. Terrones, and B. G. Sumpter, Nano Lett. 11, 3267-3273 (2011).

${ }^{39}$ Z. Qiao, S. A. Yang, B. Wang, Y. Yao, and Q. Niu, Phys. Rev. B 84, 035431 (2011).
${ }^{40}$ L. Pan, J. An, Y. J. Liu, and C. D. Gong, Phys. Rev. B 84, 115434 (2011).

${ }^{41}$ V. M. Pereira, J. M. B. Lopes dos Santos, and A. H. Castro Neto, Phys. Rev. B 77, 115109 (2008).

${ }^{42}$ O. V. Yazyev and L. Helm, Phys. Rev. B 75, 125408 (2007).

${ }^{43}$ S. Sorella and E. Tosatti, Europhys. Lett. 19, 699 (1992).

${ }^{44}$ Z. Y. Meng, T. C. Lang, S. Wessel, F. F. Assaad, and A. Muramatsu, Nature (London) 464, 847 (2010).

${ }^{45}$ K. L. Lee, B. Gremaud, R. Han, B. G. Englert, and C. Miniatura, Phys. Rev. A 80, 043411 (2009).

${ }^{46}$ M. Gibertini, A. Singha, V. Pellegrini, M. Polini, G. Vignale, A. Pinczuk, L. N. Pfeiffer, and K. W. West, Phys. Rev. B 79, 241406 (2009).

${ }^{47}$ K. K. Gomes, W. Mar, W. Ko, F. Guinea, and H. C. Manoharan, Nature 483, 306 (2012).

${ }^{48}$ L. Brey and H. A. Fertig, Phys. Rev. B 73, 235411 (2006).

${ }^{49}$ O. V. Yazyev and M. I. Katsnelson, Phys. Rev. Lett. 100, 047209 (2008).

${ }^{50}$ Ant.U (Alicante NanoTransport). U is part of the ALACANT quantum transport software package [http://alacant.dfa.ua.es/index.html]. 